\title{
Genetic Diversity Using Spoligotyping Method in the Case of Pulmonary Tuberculosis in Certain Localities of West Region of Cameroon
}

\author{
Assam Assam Jean Paul 1, 3, *, Feutsa Tegiogni Vanessa Therance, ${ }^{2,3}$ Godwe Celestin $^{3}$, \\ Doungmo Toumbou Severin ${ }^{3}$, Teuma Djeumetio Sylvain ${ }^{3}$, Penlap Beng Veronique ${ }^{2,3}$ \\ ${ }^{1}$ Laboratory of Microbiology, Faculty of Science, University of Yaoundé I, Yaoundé, Cameroon \\ ${ }^{2}$ Laboratory of Biochemistry, Faculty of Science, University of Yaoundé I, Yaoundé, Cameroon \\ ${ }^{3}$ Laboratory for Infectious Diseases Research and Pharmacology, Biotechnology Centre, University of Yaoundé I, Yaoundé, Cameroon
}

Email address:

assamjean@yahoo.fr (A. A. J. Paul)

${ }^{*}$ Corresponding author

\section{To cite this article:}

Assam Assam Jean Paul, Feutsa Tegiogni Vanessa Therance, Godwe Celestin, Doungmo Toumbou Severin, Teuma Djeumetio Sylvain, Penlap Beng Veronique. Genetic Diversity Using Spoligotyping Method in the Case of Pulmonary Tuberculosis in Certain Localities of West Region of Cameroon. Biochemistry and Molecular Biology. Vol. 5, No. 1, 2020, pp. 6-11. doi: 10.11648/j.bmb.20200501.12

Received: February 20, 2020; Accepted: March 9, 2020; Published: April 1, 2020

\begin{abstract}
Tuberculosis (TB) remains a major global health problem. In 2016, an estimated 10.4 million people developed TB and 1.8 million died from the disease. TB epidemiology in Cameroon has so far largely consisted of reporting the number of cases detected and their demographic data. The present study investigates the genetic diversity among Mycobacterium tuberculosis complex circulating in the west region of Cameroon and analyzes the relationship between genotypes and epidemiology situation of patient. Spoligotyping was performed by PCR-amplification followed by the reverse hybridization of 120 cultured specimens and were identified by comparison to reference strains in SITVIT database. Mycobacterium tuberculosis was the only species identified. LAM10_CAM and T1 families were the most prevalent genetic families with $43.33 \%$ and $31.66 \%$ respectively. No association was found between genotypes with HIV serostatus. However, there was a significant association between genotypes and the gender also between genotypes and Patients age. Patients with age above 35 were more likely infected by LAM10_CAM strains, and belong genotypes and locality where the patients of Dschang were more likely infected by T1 strain. Population structure of Mycobacterium tuberculosis complex strains from the West region of Cameroon was found to be diverse and the Share Type 61 from the LAM10_CAM family was highly predominant.
\end{abstract}

Keywords: Tuberculosis, Spoligotypage, Cameroon, Genetic Diversity

\section{Introduction}

Tuberculosis (TB) remains a major global health problem. In 2016, an estimated 10.4 million people developed TB and 1.8 million died from the disease. The case fatality ratio (the global proportion of people with TB who die from the disease) varied from under $5 \%$ in a few countries to more than $20 \%$ in most countries in the WHO African Region [1]. Cameroon is a country with high TB incidence [2] with a high prevalence in the west region (11\%) [3]. Elimination of TB by 2050 is a long-term goal of the World Health Organization (WHO) and their strategy is heavily based on the improvements in the current diagnostics, treatment and vaccination, as well as on the development of new strategies to control and fight the epidemic [4]. Any strategy for combating the epidemic should be based on a thorough appreciation of the problem. Interventions driven by a poor understanding of the pathogen in a specific geographical context will necessarily entail a high risk of failure [5]. Our understanding of the transmission of tuberculosis (TB) has been greatly enhanced since the introduction of DNA fingerprinting techniques for Mycobacterium tuberculosis [6]. Spoligotyping is a very practical and re-producible PCR-based method, which assays the presence or the absence of a set of target sequences in the direct repeat (DR) locus [7]. 
The resulting genotype has a simple binary format, which has recently leaded to the construction of large databases, intended to facilitate recognition of the origin of a particular clinical isolate [8]. Another advantage of spoligotyping is that it can be used simultaneously for the detection and typing of the M.tuberculosis complex bacteria in one assay. As in most resource poor countries, TB epidemiology in Cameroon has so far largely consisted of reporting the number of cases detected and their demographic data. Little is known about Mycobacterium tuberculosis strains circulating in the west region of the country. The aim of the present study was to identify and evaluate the genetic diversity of Mycobacterium tuberculosis complex isolates circulating in the west region of Cameroon 4 years after lhe last studies, to determine the major genotypes responsible for transmission and to analyze the relationship between genotypes and epidemiology situation of patient.

\section{Methodology}

\subsection{Study Setting and Design}

This was a cross-sectional study involving all pulmonary smear positive patients, whose age was ranged from 15 to 65 (mean age, 33.75 years) attending Foumban, Mbouda, Dschang and Bagangté District Hospitals from April to November 2017. All patients underwent physical examination and their histories were recorded. This study included 120 Mycobacterium tuberculosis complex isolates selected from smear positive pulmonary tuberculosis patients admitted in the various district hospitals. Sample processing, confirmatory microscopy and serology were done at the hospital laboratory. Culture, molecular analysis and quality control (internal and external) were performed at the Laboratory for Tuberculosis Research (LTR), Biotechnology Centre, University of Yaoundé I. A study questionnaire was designed for patient data collection at the study site including gender, age, marital status, educational level, place of residence (urban vs rural), previous incarceration and clinical data (HIV status, previous TB treatment).

\subsection{Sample Processing}

Samples were collected from each participant with productive cough on two consecutive days following standard procedures. Ziehl-Neelsen and/or auramine smear examinations $[9,10]$ were performed at the recruitment site. Only samples with the highest smear grade were transported in a cold box within one day to the LTR for confirmatory microscopy, and culture. Each specimen was submitted to a decontamination step using Cetylpyridinium chloride/ $\mathrm{NaCl}$. HIV testing was performed using a microparticle enzyme immunoassay (AxSYM HIV Ag/Ab Combo) (Abbot Laboratories) for all consenting participants. Each participant received appropriate counselling prior to blood collection.

\subsection{Sputum Culture and Identification}

After centrifugation, the sediment of the decontaminated product was used to inoculate three Lo“wenstein Jensen (LJ) media slants, of which one was supplemented with a $0.4 \%$ pyruvate. Cultures were incubated at $37^{\circ} \mathrm{C}$, read weekly for growth and considered negative when no colony was obtained after 8 weeks of incubation. Final species identification of $M$. tuberculosis was based on growth rate and colony morphology observation. The paranitrobenzoic acid (PNB) test was used to distinguish atypical mycobacteria from $M$. tuberculosis complex strains.

\subsection{DNA Extraction}

Mycobacterium tuberculosis complex were scraped from Löwenstein-Jensen slopes, collected into Eppendorf tubes containing Tris-EDTA (10 mM, $1 \mathrm{mM}, \mathrm{pH} 8)$ and heated for $30 \mathrm{~min}$ at $90^{\circ} \mathrm{C}$. After centrifugation at $13,000 \mathrm{xg}$, the supernatant was collected into a new tube and kept at $-20^{\circ} \mathrm{C}$ until further use.

\subsection{Spoligotyping}

All isolates were genotyped with a spoligotyping commercial kit (Isogen Bioscience, BV Maarsen, The Neth-erlands) according to the protocol previously described by Kamerbeek et al. [7]. Briefly, the DR region of the TB genome was amplified using primers DRa, 5'-GGTTTTGGGTCTGACGAC-3'(biotinylated5'end) and DRb, 5'-CCGA-GAGGGGACGGAAAC-3'. PCR products were hybridized with a set of 43 spacer oligonucleotides covalently linked to the spoligo membrane (Isogen Life Sciences, The Netherlands) according to the manufacturer's instructions. The hybridized PCR products were then incubated with a streptavidin-peroxidase conjugate and the membrane exposed to chemiluminescence (Amersham ECL Direct $^{\mathrm{TM}}$ nucleic acid labeling and detection system, GE Healthcare Limited, UK). The X-ray film was developed using standard photochemical procedures after 20 minutes exposure. DNA extracts of $M$. tuberculosis $\mathrm{H} 37 \mathrm{Rv}$ and $M$. bovis BCG were used as controls.

\subsection{Data Analysis}

Spoligotype patterns in a binary format were entered in an Excel sheet, and compared with the spoligotype data-base SpolDB4 using MIRU VNTR plus [11]. The Hunter Gaston Discriminatory Index (HGDI) was used to calculate the discriminatory power of spoligotyping method [12]. The Chi square or Exact Fisher test when necessary were employed to evaluate difference in serology, age group between LAM10_CAM and non LAM10_CAM strains. Values of $p$ of less than 0.05 were considered significant.

\subsection{Ethical Consideration}

This study was in accordance with the ethical standards of the institutional ethics committee. Hence, Ethical Clearance $\mathrm{N}^{\circ} 209 / \mathrm{CNE} / \mathrm{SE} / 17$ and an Administrative Authorization from each district hospital were obtained. All data collection activities were careful explained before and during the study. Signed informed written consent was obtained from each 
enrolled patient who did not incur any cost for processing of the samples.

\section{Results}

Of the 120 isolates collected from patients, 69 came from men $(65 \%)$ and 51 from women $(35 \%)$, a sex ratio of 2 in favor of the male sex. Forty-one isolates were from TB positive patients from Foumban District Hospital, 29 from Bangangté District Hospital, 25 from Mbouda District Hospital and 25 from Dschang District Hospital. 51 (42.5\%) patients were single and $69(57.5 \%)$ patients were married. HIV serology was available for 114 (95\%), among which 36 $(31.57 \%)$ were HIV positive. Of the 120 isolates analyzed, all were classified as Mycobacterium tuberculosis.

\subsection{Distribution of Different Genetic Families}

Among the 120 typed isolates, a total of 18 different profiles clustered into 5 genetic families were identified. Of these, 52 (43. 33\%) isolates belong to the LAM10_CAM family while $61(50.83 \%)$ were non LAM10_CAM strains. Strains classified into non LAM10_CAM family included strains from the Haarlem family (10.83\%), T1 family $(31.66 \%)$ and others (Table 1).

Table 1. Distribution of different genetic families identified in a collection of 298 Mycobacterium tuberculosis complex isolates.

\begin{tabular}{lll}
\hline Genetic families & No. isolates & Frequency (\%) \\
\hline LAM10_CAM & 52 & $43.33 \%$ \\
H3 & 13 & $10.83 \%$ \\
T1 & 38 & $31.66 \%$ \\
\hline
\end{tabular}

\begin{tabular}{lll}
\hline Genetic families & No. isolates & Frequency (\%) \\
\hline T5 & 2 & $1.66 \%$ \\
T2 & 8 & $6.66 \%$ \\
Non identified & 7 & $5.83 \%$ \\
\hline
\end{tabular}

\subsection{Predominant Spoligotypes}

Of the 18 spoligotypes identified, 10 were represented as Shared Types (ST) according to SpolDB4 while the others were reported for the first time. Among these Shared Types, ST 61 member of the LAM10_CAM, ST 53 member of the T1 Ghanaeen family and ST 50 member of the Haarlem family respectively represented $42 \%, 25 \%$ and $10.83 \%$ (Table 2). The LAM10_CAM family prevalence was significantly higher in men $(48.72 \%)$ and patients over 35 years of age $(54.29 \%)$ compared to women $(19.05 \%)$ and patients under 35 years of age $(16.00 \%)$. The other variables tested were not significantly associated with infection by this family. The prevalence of the T1 strain was significantly higher in Dschang $(70 \% ; p=0.027)$, the other variables tested were not significantly associated with infection by this spoligotype. The prevalence of the T2 strain was significantly high in patients under 30 years of age $(100 \%)$ and in single patients $(100 \%)$, unlike patients over 30 years of age $(0 \%)$ and married patients $(0 \%)$. other variables tested were not significantly associated with infection with this spoligotype. None of the variables tested were associated with infection by the T5 spoligotype and the H3 family (Table 3). Genetic profiles are both unknown in the database (SPOLB4) and (SITVIT WEB) was $5.83 \%$ for a clustering rate (TC) of $70 \%$, and a Hunter Gaston Discriminatory Index (HGDI) of 0.79 .

Table 2. Distribution of Share Types (ST) identified in a collection of 120 Mycobacterium tuberculosis complex isolates.

\begin{tabular}{|c|c|c|c|c|}
\hline Genotypes & Share-Types (ST) & Spoligo-patterns & No. isolates & Frequency (\%) \\
\hline \multirow{3}{*}{ LAM10_CAM } & 61 & و & 48 & $40 \%$ \\
\hline & 852 & 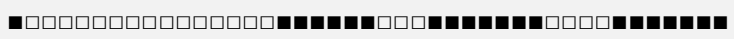 & 2 & $1.66 \%$ \\
\hline & 838 & 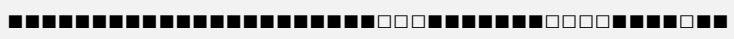 & 2 & $1.66 \%$ \\
\hline \multirow{3}{*}{$\mathrm{T} 1$} & 1129 & 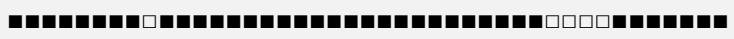 & 4 & $3.33 \%$ \\
\hline & 1166 & 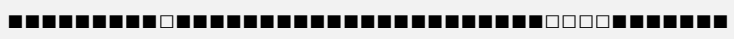 & 2 & $1.66 \%$ \\
\hline & 144 & 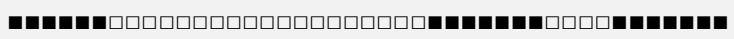 & 2 & $1.66 \%$ \\
\hline $\mathrm{T} 2$ & 52 & 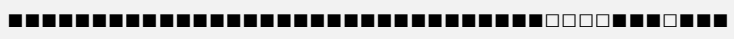 & 6 & $5 \%$ \\
\hline T5 & 44 & 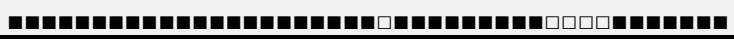 & 2 & $1.66 \%$ \\
\hline
\end{tabular}

Table 3. Correlation between different genotypes of the strains and the epidemiological parameters of the patients.

\begin{tabular}{|c|c|c|c|c|c|c|c|}
\hline \multicolumn{2}{|l|}{ Genotypes } & \multicolumn{2}{|c|}{ LAM10_CAM } & \multicolumn{2}{|l|}{ T1 } & \multicolumn{2}{|l|}{ T5 } \\
\hline Variables & Modalities & frequency & $\mathbf{P}$ & frequency & $\mathbf{P}$ & frequency & $\mathbf{P}$ \\
\hline \multirow[t]{4}{*}{ Localities } & Baganté & $15(50 \%)$ & 0.1888 & $6(21 \%)$ & 0.027 & $0(0)$ & 0.7221 \\
\hline & Dschang & $3(10 \%)$ & & $18(70 \%)$ & & $0(0)$ & \\
\hline & Foumban & $15(38 \%)$ & & $9(23 \%)$ & & $2(3.84 \%)$ & \\
\hline & Mbouda & $13(50 \%)$ & & $5(20 \%)$ & & $0(0)$ & \\
\hline \multirow[t]{2}{*}{ gender } & Female & $10(19.05 \%)$ & 0.0242 & $34(66.66 \%)$ & 0.6793 & $2(4.76 \%)$ & 0.1694 \\
\hline & Male & $34(48.72 \%)$ & & $19(28.2 \%)$ & & $0(0)$ & \\
\hline Age (year) & $>35$ & $32(54.29 \%)$ & & $14(22.85 \%)$ & & $2(2.85 \%)$ & \\
\hline \multirow[t]{2}{*}{ Marital Statut } & Single & $15(28.57 \%)$ & 0.2538 & $17(33.33 \%)$ & 0.6793 & $0(0)$ & 0.4593 \\
\hline & Maried & $40(58.6 \%)$ & & $19(28.2 \%)$ & & $2(2.56 \%)$ & \\
\hline \multirow[t]{3}{*}{ Level of study } & None & $12(45.5 \%)$ & 0.6735 & $7(27.27 \%)$ & 0.6771 & $0(0)$ & 0.4624 \\
\hline & Primary & $14(47.05)$ & & $4(14.81 \%)$ & & $2(5.88 \%)$ & \\
\hline & Secondary & $10(33.33 \%)$ & & $8(27.77 \%)$ & & $0(0)$ & \\
\hline
\end{tabular}




\begin{tabular}{|c|c|c|c|c|c|c|c|}
\hline \multicolumn{2}{|l|}{ Genotypes } & \multicolumn{2}{|c|}{ LAM10_CAM } & \multicolumn{2}{|l|}{ T1 } & \multicolumn{2}{|l|}{ T5 } \\
\hline Variables & Modalities & frequency & $\mathbf{P}$ & frequency & $\mathbf{P}$ & frequency & $\mathbf{P}$ \\
\hline \multirow{3}{*}{ Residence } & Higher & $10(28.57 \%)$ & & $15(42.85 \%)$ & & $0(0)$ & \\
\hline & Rural & $19(33.33 \%)$ & 0.6646 & $19(33.33 \%)$ & 0.4902 & $2(2.77 \%)$ & 0.4103 \\
\hline & Urban & $26(41.16 \%)$ & & $16(25 \%)$ & & $0(0)$ & \\
\hline \multirow{3}{*}{ HIV Statut } & Inconnu & $4(60 \%)$ & 0.1114 & $2(30 \%)$ & 0.5995 & $0(0)$ & 0.745 \\
\hline & Négatif & $31(39.47)$ & & $21(26.31 \%)$ & & $2(2.63 \%)$ & \\
\hline & Positif & $6(16.66 \%)$ & & $15(41.66 \%)$ & & $0(0)$ & \\
\hline
\end{tabular}

Table 3. Continued.

\begin{tabular}{|c|c|c|c|c|c|}
\hline Genotypes & & T2 & & H & \\
\hline Variables & Modalities & frequency & $\mathbf{P}$ & frequency & $\mathbf{P}$ \\
\hline \multirow[t]{4}{*}{ Localities } & Baganté & $0(0)$ & 0.2022 & $0(0)$ & 0.1888 \\
\hline & Dschang & $3(10 \%)$ & & $0(0)$ & \\
\hline & Foumban & $5(11.53 \%)$ & & $3(7.69 \%)$ & \\
\hline & Mbouda & $0(0)$ & & $5(20 \%)$ & \\
\hline \multirow[t]{2}{*}{ gender } & Female & $2(4.76 \%)$ & 0.6643 & $7(14.28 \%)$ & 0.0825 \\
\hline & Male & $5(7.69 \%)$ & & $2(2.5 \%)$ & \\
\hline \multirow[t]{2}{*}{ Age (year) } & $\leq 35$ & 0 & & 0 & \\
\hline & $>35$ & $10(16 \%)$ & 0.0259 & $7(12 \%)$ & 0.2984 \\
\hline \multirow[t]{2}{*}{ Marital Statut } & Single & $0(0)$ & & $1(2,85 \%)$ & \\
\hline & Maried & $13(19.04 \%)$ & 0.0048 & $3(4.76 \%)$ & 0.6643 \\
\hline \multirow[t]{4}{*}{ Level of study } & None & $0(0)$ & & $2(8.33 \%)$ & \\
\hline & Primary & $0(0)$ & 0.1794 & $0(0)$ & 0.6738 \\
\hline & Secondary & $0(0)$ & & $4(11.76 \%)$ & \\
\hline & Higher & $6(16.66 \%)$ & & $2(5.5 \%)$ & \\
\hline \multirow[t]{2}{*}{ Residence } & Rural & $4(7.14 \%)$ & & $4(7.14)$ & \\
\hline & Urban & $2(2.7 \%)$ & 0.1391 & $2(2.7 \%)$ & 0.1391 \\
\hline \multirow[t]{3}{*}{ HIV Statut } & Inconnu & $0(0)$ & 0.6505 & $0(0)$ & 0.2892 \\
\hline & Négatif & $6(7.89 \%)$ & 0.2022 & $8(10.52 \%)$ & \\
\hline & Positif & $3(8.33 \%)$ & & $0(0)$ & \\
\hline
\end{tabular}

\section{Discussion}

It has been reported in some instances that, spoligotyping can distinguish among members of the M. tuberculosis complex based on the species-specific presence/absence of spacers [11]. In our study, all species were identified as $M$. tuberculosis. A similar observation was reported in previous work among the M. tuberculosis complex isolates collected from the West region of Cameroon [12]. The gender distribution of positive microscopy patients recorded has been noted here, as it already mentioned by several studies carried out around the world, which indicate that men are more affected by pulmonary tuberculosis than women [1]. Several scientific publications show the predisposition of male patients to contract bacilli of the Mycobacterium tuberculosis complex $[13,14]$ compared to female patients. This seems to be due to certain specific factors generally linked to men's lifestyle [15].

Research on the genetic polymorphism of strains of the $M$. tuberculosis complex in humans in Cameroon has shown that M. tuberculosis is the predominant agent in tuberculosis cases [16]. The species M. africanum does not appear in our result indicating a shift of this species compared to the work published in the 1970s where several cases of tuberculosis reported were due to $M$. africanum (56\%) [17]. This regression of $M$. africanum, as an etiological agent of tuberculosis in Cameroon, over the past 4 decades, was already mentioned by Niobe-Eyangoh et al., (2003) and by
Assam (2012) [12, 13]. A similar phenomenon had already been noted in other African countries, such as in Burkina Faso $[18,19]$. Although the factors that can justify this change in etiological agent are not yet well known, the treatment protocol currently applied could undoubtedly play an essential role [19].

The spoligotyping results also revealed an absence of spoligotype corresponding to the species $M$. bovis, as described by studies conducted in Cameroon by Niobe Eyangoh et al., (2003) [13], and carried out in 2004 in Burkina-Faso by Easterbrook et al., (2004) [20].

The non-involvement of $M$. bovis in the onset of pulmonary tuberculosis is due to several factors, namely: the major responsibility for $M$. bovis in cases of extra-pulmonary tuberculosis not taken into account in our study [19]; the highest frequency of pulmonary tuberculosis caused by $M$. bovis in rural areas [19], but the majority of our patients came from urban areas (Foumban, Bangangté, Mbouda, Dschang) and the small sample size.

Research of the genetic profiles of the 120 isolates in the database of international spoligotypes (SpolDB4) and SITVIT WEB enabled us to identify 5 families of spolygotypes LAM10_CAM (43.33\%); 11 (31.66\%); Haarlem (10.83\%); T2 $(6.66 \%)$; T5 (1.66\%). The family mainly represented being the LAM10 CAM family $(43.33 \%)$ against $30.98 \%$ of LAM10_ CAM found by Assam (2012) [13]. These results corroborate with those found by Niobe who had obtained a prevalence of $46.73 \%$ for the LAM 10 _ CAM family in the same region [12]. The $\mathrm{T} 1$ family is the second family mostly 
represented with a prevalence of $31.66 \%$, within this family the most represented clade is the site ST 53 or Ghana family with a prevalence of $25 \%$ compared to all our isolates. The high prevalence of LAM10_CAM, and T1 families noted in our study shows that these families, would spread quickly but also reflect a gene mutation in the DR region on the other part (Assam, 2012) [13]. Also, we noted an increase prevalence of isolates belonging to the Share Type ST 53 (25\%), compared to $16.9 \%$ found by Assam Assam (2012) [13] and 4.5\% found by Niobe-Eyangoh et al.., (2003) [12]; This indicates a strong infiltration of the Ghana family in the past 12 years in west Cameroon, probably due to migration phenomena. We also noted an increase of genotypes Haarlem family (10.83\%) compared to $4.2 \%$ found by Assam Assam et al., (2012) [13] and a reduction of $10 \%$ compared to the studies done by Koro Koro et al., (2013) [21] who found that the Haarlem family was around $18 \%$ in the same region. The T2 family $(6.66 \%)$ remained constant compared to the results of Koro Koro et al., (2013)[21] who found that the T2 family was around 7\%.

Species identification tests from positive cultures showed a large predominance of the $M$. tuberculosis species with an absence of $M$. africanum and $M$. bovis. All the observations made above allow us to confirm that the incidence of tuberculosis in Cameroon remains high, and to maintain that one of the important factors responsible for this increase could be correlated with the different epidemiological parameters of patients. The prevalence of the LAM10_CAM strain was significantly higher in men $(p<0.05)$ and in patients over 35 years of age $(\mathrm{p}<0.05)$ compared to women and patients under 30 years of age. This could be explained by the fact that this family would have adapted to the lifestyle of patients over 35 years old and to the habits of men. These results corroborate those of Kamgue et al. at the centre, which found that patients belonging to the 35-44 age group were significantly more likely to be infected with the LAM10_CAM strain [14]. The prevalence of the T1 strain was significantly higher in Dschang (70\%; p 0.027). This could be explained by the fact that this strain would have adapted to the climate and lifestyle of the inhabitants of this locality. The prevalence of the T2 strain was significantly high in patients under 35 years of age $(p<0.05)$ and in singles $(p<0.05)$ in contrast to patients over 35 years of age and married couples. This could be explained by the adaptation of this strain to the lifestyle of young people.

\section{Conclusion}

It emerges from this study that:

The population structure of Mycobacterium tuberculosis complex strains from the west region was diverse and very dynamic compared to previous studies in the same region. It included 18 different genotypes. The majority of strains belonged to the LAM10_CAM which can be subdivided in 3 spoligotypes. The consequence of this diversity for the TB epidemic are not yet clear and need to be addressed in further studies

\section{References}

[1] WHO: Global tuberculosis report. In. Edited by WHO/HTM/TB/2016.13. 20 Avenue Appia, 1211 Geneva 27, Switzerland World Health Organization; 2016.

[2] S. Kwedi Nolna, I. D. Kammogne, R. Ndzinga, B. Afanda, R. Ntone, Y. Boum Y, D. Nolna: Community knowledge, attitudes and practices in relation to tuberculosis in Cameroon. The international journal of tuberculosis and lung disease: the official journal of the International Union against Tuberculosis and Lung Disease 2016, 20 (9): 1199-1204.

[3] Program NTC: [Strategic plan for the fight against tuberculosis in Cameroon, 2015-2019]. In.; 2015: 1-74.

[4] World Health Organization, "Global Tuberculosis Control: WHO Report 2011,’Geneva, 2011.

[5] G. D. Van der Spuy, R. M. Warren and P. D. Van Helden, "The Role of Molecular Epidemiology in Low-Income, High-Burden Countries," International Journal of Tuber-cle and Lung Diseases, Vol. 13, No. 4, 2009, pp. 419-420.

[6] D. Van Soolingen, P. W. Hermans, P. E. De Haas, D. R. Soll and J. D. Van Embden, "Occurrence and Stability of Insertion Sequences in Mycobacterium tuberculosis Com-plex Strains: Evaluation of an Insertion Sequence-De-pendent DNA Polymorphism as a Tool in the Epidemiol-ogy of Tuberculosis," Journal of Clinical Microbiology, Vol. 29, No. 11, 1991, pp. 2578-2586.

[7] J. Kamerbeek, L. Schouls, A. Kolk, M. Van Agterveld, D. Van Soolingen, S. Kuijper, A. Bunschoten, H. Molhuizen, R. Shaw, M. Goyal, et al., "Simultaneous Detection and Strain Differentiation of Mycobacterium tuberculosis for Diagnosis and Epidemiology," Journal of Clinical Mi-crobiology, Vol. 35, No. 4, 1997, pp. 907-914. doi: 10.3201/eid0811.020125.

[8] I. Filliol, J. R. Driscoll, D. Van Soolingen, B. N. Kreis-wirth, K. Kremer, G. Valetudie, D. D. Anh, R. Barlow, D. Banerjee, P. J. Bifani, et al., "Global Distribution of My-cobacterium tuberculosis Spoligotypes," Emerging Infec-tious Diseases, Vol. 8, No. 11, 2002, pp. 1347-1349.

[9] J. Degommier (1957) Nouvelle technique de coloration des bacilles tuberculeux pour la recherche en fluorescence. Ann Inst Pasteur: 692-694.

[10] C. Truffot-Pernot (1990) Mycobacteries. In: Leminor L, Veron M, editors. Bacteriologie médicale. Paris: Flammarion. 965-1117.

[11] B. Mathema, N. E. Kurepina, P. J. Bifani and B. N. Krei-swirth, "Molecular Epidemiology of Tuberculosis: Cur-rent Insights," Clinical Microbiology Review, Vol. 19, No. 4, 2006, pp. 658-685. doi: 10.1128/CMR.00061-05.

[12] S. N. Niobe-Eyangoh, C. Kuaban, P. Sorlin, P. Cunin, J. Thonnon, C. Sola, et al., "Genetic Biodiversity of Myco-bacterium tuberculosis Complex Strains from Patients with Pulmonary Tuberculosis in Cameroon," Journal of Clinical Microbiology, Vol. 41, No. 6, 2003, pp. 2547-2553. doi: 10.1128/JCM.41.6.2547-2553.2003.

[13] J. P. Assam Assam. (2012). Etude de la résistance et de la diversité génomique dans le cas de la tuberculose pulmonaire au Cameroun: Régions du Centre, Ouest, Sud et Est. Thèse de Doctorat Ph. D en Biochimie, Faculté des Sciences; Université de Yaoundé I. P138. 
[14] L. Kamgue, E. Mouafo, C. Kuaban, J. Assam, J. Tedom, \& S. Niemann (2013) Estimates of genetic variability of Mycobacterium tuberculosis complex and its association with drug resistance in Cameroon. Advances in Infectious Diseases. 3: 55-59.

[15] P. Godfrey, P. Sonnenberg, S. Shearer, M. Bruce, C. Mee. \& L. Morris (2000). Tuberculosis control and molecular epidemiology in a South African. gold-mining community. Lancet. 356: 1066-1071.

[16] V. Titanji \& J. P. Assam (2016). Drug sensitivity and molecular diversity of $M$. tuberculosis in Cameroon: A Meta-analysis. Journal of Vascular Medecine \& Surgery. 4: 23-166P.

[17] M. Huet, N. Rist, G. Boube. \& D. Potier (1971). Etude bacteriologique de la tuberculose au Cameroun. Revue of Tuberculosis Pneumology. 35: 413-426.

[18] F. Simonet, M. Menard, G. Le Mao \& J. Albert (1989). Action antituberculeuse au Burkina faso. L'expérience du service de Pneumophtisiologie de l'Hôpital National Sanon Souro de Bobo-Dioulasso. Societé Santé Industrie Service, Paris, France.

[19] M. Vekemans, M. Cartoux, S. Diagbouga, M. Dembele, B. Kone, A. Delafosse, A. Dera \& P. Van De Perre (1999). Potential source of human exposure to Mycobacterium bovis in Burkina Faso, in the context of the HIV epidemic. Clinical Microbiology of Infection. 5: 617-621.

[20] P. Easterbrook, A. Gibson, S. Murad, D. Lamprecht, N. Ives, A. Ferguson, O. Lowe, P. Mason, A. Ndudzo \& A. Taziwa (2004). High rates of clustering of strains causing tuberculosis in Harare, Zimbabwe: a molecular epidemiological study. Journal of clinical Microbiology. 42: 4536-4544.

[21] F. Koro Koro, Y. Kamdem Simo, F. Piam, C. Kuaban, \& S Eyangoh (2013). Population dynamics of tuberculous bacilli in Cameroon as assessed by spoligotyping. Journal of Clinical Microbiology. 51: 299-302. 